\title{
1.5-dimensional simulation of free electron maser amplifiers
}

\author{
A. V. Elzhov, ${ }^{*}$ A. K. Kaminsky, E. A. Perelstein, S. N. Sedykh, and A. P. Sergeev \\ Joint Institute for Nuclear Research, Dubna 141980, Russia \\ (Received 28 December 2004; revised manuscript received 26 July 2005; published 26 September 2005)
}

A 1.5-dimensional model of Compton/Raman free-electron laser (FEL) amplifiers with helical wigglers has been composed by using the helical-trajectory approach. This model has been used to analyze results of earlier experimental investigations of Compton/Raman millimeter-wavelength FEL amplifiers. The results of the $1.5 \mathrm{D}$ simulation are in reasonable agreement with $3 \mathrm{D}$ simulation and experimental results obtained in the regimes with reversed and zero guide magnetic fields. Thus a convenient tool has been developed to determine basic amplification parameters for possible experiments in the future.

DOI: $10.1103 /$ PhysRevSTAB.8.090701

PACS numbers: 29.27.-a, 29.85.+c, 29.17.+w

\section{INTRODUCTION}

A large number of papers is devoted to investigate freeelectron laser (FEL) amplifiers. The basic theoretical material can be found in [1-4]. Experimental investigations have been carried out as well. In particular, results of some experiments in the millimeter- and submillimeterwavelength range are reported in [5-15] (see also references therein). In the majority of those papers, the experimental results are compared with calculated data. The problems of numerical simulation are discussed specifically in [16-19]. The last papers also include a basic bibliography.

A full-scale three-dimensional simulation $[17,18]$ allows one to get the best agreement between the simulation and experimental data. However, relatively simple approximate models seem to be attractive to use if they take dominating physical processes into account and allow one to determine the characteristics of an amplifier with an acceptable accuracy.

In this paper, we compose a 1.5-dimensional model upon the helical-trajectory approach where the transverse motion of the electrons is described only with the transverse velocity amplitude. Variation of this transverse velocity amplitude due to interaction with the radiation field is a distinctive feature of the $1.5 \mathrm{D}$ model and results in the changing of the beam-wave coupling factor. This model is applied to analyze various regimes of the millimeter FEL [free-electron maser (FEM)] amplifier with respect to the space-charge effects (Compton/Raman regimes) as well as to the axial guide magnetic field. These theoretical results are compared with experiments $[7,11,14]$. The applicability of the model used is verified via comparison with the known 3D simulations.

The developed code is considered to be a convenient tool to evaluate a possible experiment with a FEM amplifier in the future. Besides, it is of practical importance for studying the features of the FEM oscillator, which has already been elaborated at JINR together with IAP RAS (Nizhny

\footnotetext{
*Corresponding author.

Electronic address: artel@sunse.jinr.ru
}

Novgorod) during the past ten years [20,21]. It consists of a helical wiggler, a solenoid producing the reversed guide magnetic field, and a Bragg resonator providing the selective feedback.

\section{FEL AMPLIFIER 1.5D MODEL}

Let us study the self-consistent spatial problem of a relativistic electron beam moving in the combined electromagnetic field consisting of the external magnetic field produced by the wiggler and the solenoid and the microwave field. We assume that the regime is stationary, the wave is single-mode, and its amplitude varies slowly with length. The space-charge effects as well as the beam energy spread are taken into account.

On the role of space-charge effects, there are two extreme types of FEL operation: high-gain Compton and Raman regimes.

The Raman regime of the FEL amplifier, where collective effects dominate, is valid under three conditions [17]:

(1) The system is long enough; i.e., the beam is sufficiently dense so that several plasma oscillations are executed when propagating through the wiggler:

$$
\lambda_{p} / L \leq 1 \text {. }
$$

Here $\lambda_{p}=2 \pi c / \omega_{p}, \omega_{p}$ is the relativistic plasma frequency $\omega_{p}^{2}=4 \pi n_{e} e^{2} / m_{0} \gamma_{z}^{2} \gamma_{0}$, where $n_{e}$ is the electron beam density, $m_{0}$ is the electron rest mass, and $\gamma_{0}$ and $\gamma_{z}$ are the total and longitudinal relativistic factors.

(2) The transverse electron velocities are much less than the critical value [4]

$$
\beta_{w} \ll \beta_{\text {crit }} \equiv F^{-1 / 2}\left(2 \omega_{p} c^{2} / \nu_{z 0}^{3} \gamma_{z}^{2} k_{w}\right)^{1 / 2} .
$$

Here $k_{w}=2 \pi / \lambda_{w}$ is the wiggler wave number ( $\lambda_{w}$ is the wiggler period), $\nu_{z 0}$ is the initial longitudinal electron velocity, and $F$ is the filling factor close to the ratio between the electron beam and waveguide cross sections.

This criterion is based on comparison of the contributions of the intrinsic beam and external fields, and is obtained by using the helical-trajectory approach. When analyzing real experiments, one should apply the criterion 
carefully since [17] (1) the boundary conditions at the waveguide surface reduce the effective plasma frequency and (2) the bulk characteristics of the electron orbits are modified by wiggler inhomogeneities, beam thermal effects, and use of an axial guide magnetic field. Because of these complications, the helical model may be insufficient to describe space-charge effects, and a full 3D nonlinear analysis is often needed.

(3) Landau damping of the space-charge waves is not significant when the space-charge wavelength is greater than the Debye length. The restriction on the Raman amplification is imposed by the beam energy spread. Landau damping is negligible for a Gaussian distribution of electron velocities if [2]

$$
(\delta \gamma / \gamma)_{z}<\left(\lambda_{w} / 2 \gamma\right)\left(\omega_{p} / 2 c\right) \equiv \pi \lambda_{w} / 2 \gamma \lambda_{p} .
$$

Assume the electron orbits are helical trajectories with transverse velocity $\beta_{\perp}$; then a set of equations can be written as follows for a FEL including space charge $[9,22,23]$ :

$$
\begin{gathered}
\frac{d \gamma_{j}}{d Z}=i \frac{\beta_{\perp j}}{2 \beta_{z j}} a_{s} e^{i \psi_{j}} \\
+\frac{c}{4 \omega_{0}} \frac{\omega_{p}^{2} \gamma_{z 0}^{2} \Phi_{0} \beta_{z j} F_{\mathrm{sc}}}{c^{2}\left(k_{z}+k_{w}\right)} i e^{i \psi_{j}}\left\langle e^{\left.-i \psi_{i}\right\rangle}+\right.\text { c.c., } \\
\frac{d \theta_{j}}{d Z}=\frac{1}{\beta_{\mathrm{ph}}}-\frac{1}{\beta_{z j}} \\
\frac{d \hat{a}}{d Z}=i \eta\left\langle\frac{\beta_{\perp j}}{\beta_{z j}} e^{-i \theta_{j}}\right\rangle
\end{gathered}
$$

Here $\gamma_{j}$ is the $j$ th electron relativistic factor; $Z=z \omega_{0} / c$ is the dimensionless longitudinal coordinate; $\omega_{0}=2 \pi f_{0}$ is the microwave frequency (amplifier operating frequency); $k_{z}$ is the longitudinal wave number; $\theta_{j}=k_{z} z_{j}-\omega_{0} t_{j}$ is the $j$ th electron phase relative the electromagnetic field; $\varphi$ is the phase of the complex microwave amplitude $(\hat{a}=$ $\left.a_{s} e^{i \varphi}\right) ; \psi_{j}=\varphi+\theta_{j}$ is the total ponderomotive phase. The brackets $\langle\cdots\rangle$ denote the average over the ponderomotive wavelength. The variable $a_{s}=e E_{s} / m_{0} \omega_{0} c$ is the dimensionless amplitude of the microwave electric field. The gain factor is $\eta=\left(I_{b} / I_{A}\right)(2 / N)$, where $I_{A}$ is the Alfvén current constant $\left(m_{0} c^{3} / e \approx 17 \mathrm{kA}\right)$ and $N$ is the wave norm [23] defined by the wave transverse structure. The values $\beta_{z j}$ and $\beta_{\mathrm{ph}}=\omega_{0} / c\left(k_{z}+k_{w}\right)$ are the longitudinal electron velocity and the phase velocity of the ponderomotive wave, respectively. The space-charge reduction factor $F_{\text {sc }}$ is close to the ratio of the beam and waveguide cross sections when requirement (3) is satisfied.

The constant $\Phi_{0}$ in Eq. (4) describes the effect of the presence of an axial guide magnetic field and is given by

$$
\begin{aligned}
\Phi_{0} & =1-\frac{\tilde{\beta}_{w}^{2} \gamma_{z}^{2} \Omega_{0}}{\left(1+\tilde{\beta}_{w}^{2}\right) \Omega_{0}-k_{w} c \beta_{z 0}} \\
& \equiv 1-\frac{\tilde{\beta}_{w}^{2} \gamma_{z}^{2}}{1+\tilde{\beta}_{w}^{2}-\gamma_{0} \beta_{z 0} / a_{g}},
\end{aligned}
$$

where $a_{g}=e B_{g} / m_{0} c^{2} k_{w}=\Omega_{0} \gamma_{0} / c k_{w}$ is the dimensionless strength of the guide magnetic field with the induction value of $B_{g}$, and $\tilde{\beta}_{w}=\beta_{\perp} / \beta_{z 0}$ is the generalized wiggling parameter.

To take into account the beam energy spread, we divide the beam into groups of macroparticles with equal energies. The motion of each group is defined by the electromagnetic and space-charge fields. The excitation of this field results from the contribution of each particle to the radiation. So, we employ the system such as (4)-(6) for each group of macroparticles. The initial conditions of the net system (4)-(6) include the energy distribution of the injected beam.

To apply the system (4)-(6) for simulation of the highgain Compton FEL, one can neglect the second term on the right-hand side of (4).

To determine the velocity components in Eqs. (4) and (6), it is necessary to consider the particle dynamics. In the combined magnetic field produced by a helical wiggler and a solenoid, the bulk motion of the electrons is described with equilibrium orbits [24]. The electron orbit stability with respect to the betatron oscillation excitation takes place in two branches of beam parameters and external fields. These branches are separated by the vicinity of the cyclotron resonance. For instable orbits the excitation of transversal oscillations results in their exponential growth, and the helical-trajectory approach, which assumes $\beta_{\perp} / \beta_{z} \ll 1$, breaks down.

In a real FEL the electromagnetic wave and spacecharge field influence the particle motion. If the amplitudes of induced fields, i.e., of the wave and beam, are small quantities relative the external magnetic field, then we can speak of a correction for the motion over nonperturbed stationary trajectories. Thus, the velocity vector of a particle is resolved to "unperturbed" and "perturbed" parts: $\mathbf{v}=\mathbf{v}^{0}+\delta \mathbf{v}$ (the particle index $j$ is omitted for simplicity).

The velocity of nonperturbed helical motion [24] is $\mathbf{v}^{0}=$ $\left(c \beta_{\perp 0} \cos k_{w} z, c \beta_{\perp 0} \sin k_{w} z, c \beta_{z 0}\right)$, where the amplitudes $\beta_{\perp 0}$ and $\beta_{z 0}$ can be determined from the equation for a helical equilibrium orbit:

$$
\beta_{0} \frac{\kappa_{0}^{2}}{\sqrt{1+\kappa_{0}^{2}}}=\frac{a_{g}}{\gamma_{0}} \kappa_{0}^{2}+\frac{a_{w}}{\gamma_{0}}\left(1+\kappa_{0}^{2}\right) I_{1}\left(\kappa_{0}\right),
$$

where $\kappa_{0}=\beta_{\perp 0} / \beta_{z 0}$ and $I_{1}$ is the first order modified Bessel function of the first kind. Given $\beta_{0}, \gamma_{0}, a_{g}$, and $a_{w}$, solving Eq. (7) yields $\kappa_{0}$ and the velocity components are then given by $\beta_{z 0}=\beta_{0} / \sqrt{1+\kappa_{0}^{2}}, \quad \beta_{\perp 0}=$ $\beta_{0} \kappa_{0} / \sqrt{1+\kappa_{0}^{2}}$. 
The perturbation is introduced as small changes in longitudinal and transverse velocities, $\delta \beta_{z}$ and $\delta \beta_{\perp}$, respectively. The equation for longitudinal momentum of particles will result in

$$
\begin{aligned}
\gamma \frac{d \delta \beta_{z}}{d Z}= & \frac{\beta_{\perp}}{\beta_{z 0}}\left(\frac{c k_{s}}{\omega_{0}}+\frac{d \varphi}{d Z}\right) a_{s} \sin \psi-\frac{\beta_{\perp}}{\beta_{z 0}} \frac{d a_{s}}{d Z} \cos \psi \\
& +\frac{1}{\beta_{z 0}}\left(\frac{d \gamma}{d Z}\right)_{p}-\delta \beta_{z} \frac{d \gamma}{d Z}
\end{aligned}
$$

where $(d \gamma / d Z)_{p}$ is the potential term of Eq. (4) (the second term on the right-hand side).

If $\delta \beta_{z}$ is defined, the $\delta \beta_{\perp}$ value can be calculated from the kinematic relation $\beta_{\perp}^{2}+\beta_{z}^{2}=\beta^{2} \equiv 1-\gamma^{-2}$. Then the ratios of velocity components in Eqs. (4) and (6) can be computed as $\beta_{\perp} / \beta_{z}=\left(\beta_{\perp 0}+\delta \beta_{\perp}\right) /\left(\beta_{z 0}+\delta \beta_{z}\right)$.

The assumption that the electron motion can be described by slightly perturbed helical trajectories is valid as long as the following requirements are fulfilled: (1) The azimuthal motion should lie mainly within helical trajectories; the deviations of a small scale only are allowed. (2) The particle-wave synchronism should not alter significantly; i.e., the correction for the right-hand part value of Eq. (5) should be less than that value itself. It follows from the first requirement that the azimuthal angle advance for a wiggler period should be much less than $2 \pi$. Then using the equation for azimuthal motion from [25] we get

$$
\left|\delta \beta_{\perp}\right| / \beta_{\perp 0} \ll 1 .
$$

The second requirement yields

$$
\left|\delta \beta_{z}\right| \leq 1 / 2 \gamma^{2} .
$$

\section{FEM AMPLIFIER EXPERIMENTS AND SIMULATION RESULTS}

To test the developed model, we have considered the experiments described in $[7,11,14,15]$, which cover millimeter-wave FELs (FEMs) operating in different regimes - with respect to the space charge and the guide magnetic field. The basic parameters of the FEMs are shown in Table I.

\section{A. FEM amplifier with guide magnetic field at MIT}

A detailed investigation of the FEM amplifier with a guide magnetic field was carried out at Massachusetts Institute of Technology (MIT) [11,12]. Three basic regimes were studied: (I) positive (conventional-direction) guide magnetic field less than the value corresponding to the cyclotron resonance; (II) positive guide field at the other side from the cyclotron resonance; (III) negative (reversed) field (see Table I). The experimental results indicated that the reversed field regime turned out to be most preferable with respect to transported beam current, growth rate of the of microwave power, saturation level, and energetic efficiency.

According to the criteria (1)-(3), the space-charge effect

\begin{tabular}{|c|c|c|c|c|c|}
\hline & \multicolumn{3}{|c|}{ MIT $[11,12]$} & \multirow[t]{2}{*}{ CESTA $[14,15]$} & \multirow[t]{2}{*}{ JINR [7] } \\
\hline & I & II & II & & \\
\hline Electron beam energy $E_{b}, \mathrm{MeV}$ & 0.75 & 0.75 & 0.75 & 2.2 & 1.5 \\
\hline Electron current in wiggler $I_{b}$, A & $90-119$ & 300 & 300 & 500 & $50-70$ \\
\hline Electron beam radius $r_{b}, \mathrm{~cm}$ & 0.25 & 0.25 & 0.25 & 0.5 & $0.3-0.5$ \\
\hline Initial energy spread of the electron beam, \% & $<1.5$ & $<1.5$ & $<1.5$ & $<1.5$ & $1-2$ \\
\hline Wiggler period $\lambda_{w}, \mathrm{~cm}$ & 3.18 & 3.18 & 3.18 & 12 & 7.2 \\
\hline Wiggler length $L, \mathrm{~cm}$ & 159 & 159 & 159 & 288 & 216 \\
\hline Waveguide radius, $\mathrm{cm}$ & 0.51 & 0.51 & 0.51 & 1.95 & $\sim 1.5$ \\
\hline Wiggler magnetic field strength $B_{w}$, kGs & 0.63 & 1.47 & 0.63 & 1.1 & 2.1 \\
\hline Guide magnetic field strength $B_{g}, \mathrm{kGs}$ & 4.06 & 10.92 & -10.92 & No & -1.4 \\
\hline Microwave frequency $f_{0}=\omega_{0} / 2 \pi, \mathrm{GHz}$ & 33.9 & 33.9 & 33.9 & 35 & 35 \\
\hline Radiation spectral band (FWHM), GHz & $<0.16$ & $<0.16$ & $<0.16$ & 0.16 & $0.2-0.3$ \\
\hline Operating mode (cylindrical waveguide) & $T E_{11}$ & $T E_{11}$ & $T E_{11}$ & $T E_{11}$ & $T E_{11}$ \\
\hline Power at the wiggler entrance $P_{\text {in }}, \mathrm{kW}$ & 8.5 & 8.5 & 8.5 & $8-10$ & $6-20$ \\
\hline Power at the wiggler exit $P_{\text {out }}$, MW & 5.8 & 4.2 & 61 & 15 & $2-3$ \\
\hline FEL amplifier efficiency, \% & 9 & 2 & 27 & 1.5 & $2-3$ \\
\hline Spatial increment, $\mathrm{dB} / \mathrm{m}$ & 44 & 38 & 41 & 33 & 23 \\
\hline Saturation length, $\mathrm{cm}$ & $\sim 120$ & $\sim 140$ & $\sim 160$ & $\sim 150$ & $\sim 120$ \\
\hline$\lambda_{p} / L$ ( $L$ taken equal to saturation length) & $\sim 0.20$ & $\sim 0.12$ & $\sim 0.14$ & $\sim 3.8$ & $\sim 0.46$ \\
\hline$\beta_{w} / \beta_{\text {crit }}$ & $\sim 0.45$ & $\sim 0.40$ & $\sim 0.17$ & $\sim 1.3$ & $\sim 0.57$ \\
\hline$\pi \lambda_{w} / 2 \gamma \lambda_{p}$ & $\sim 0.064$ & $\sim 0.10$ & $\sim 0.094$ & $\sim 0.0032$ & $\sim 0.038$ \\
\hline
\end{tabular}
is essential for each regime. So we deal with a definite Raman FEM amplifier especially in the regime with the

TABLE I. Parameters of FEM amplifiers. 


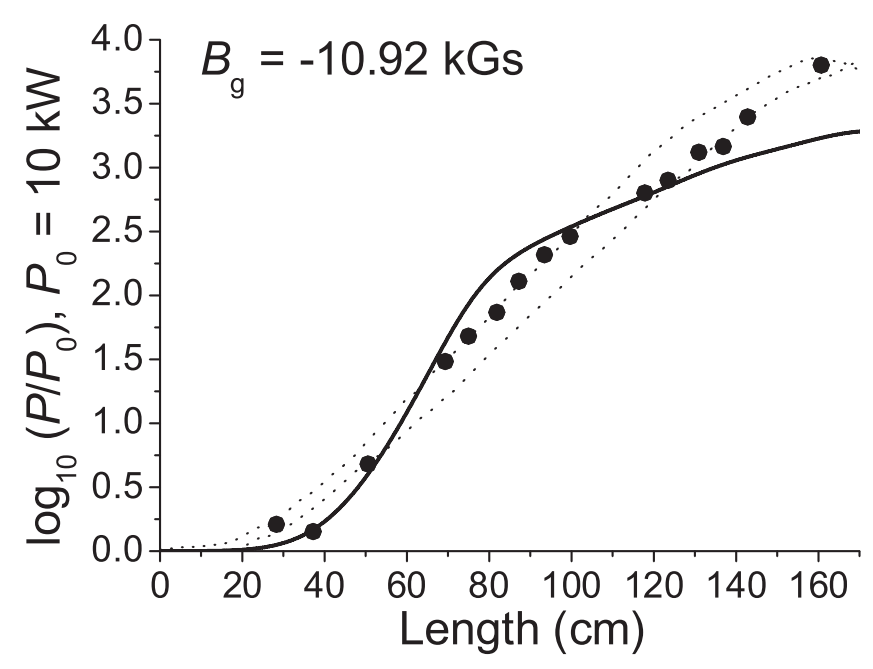

FIG. 1. Power of the FEM amplifier [11,12] versus length: experimental data (dots), simulation results from 3D code ARACHNE [16] (dotted lines for two sets of parameters within uncertainties imposed by the experiment: the lower curve for the parameters listed in Table I; the upper one-for $I_{b}=330 \mathrm{~A}$, $B_{w}=1.55 \mathrm{kGs}, P_{i n}=10 \mathrm{~kW}$ ), and simulation results from 1.5D code at the parameters of the experiment (solid line).

reversed guide field. The simulations have been performed by solving Eqs. (4)-(6) and (8) for all three regimes.

At the smaller positive guide field (case I in Table I) the operating point is relatively near the cyclotron resonance. Transversal oscillations of the particles reach such big values that the helical-trajectory approach is not applicable, and the solutions of the 1.5D model become nonconvergent. In comparison, a 3D simulation with the code ARACHNE [16] (model described in [25]) yields convergent solutions. Nevertheless, a noticeable overestimation of the growth rate and power saturation level is shown when the measured energy spread is used in the simula- tions. To put these parameters in reasonable agreement with the experiment, it is necessary to introduce an energy spread larger by several times in comparison with the measured one.

In the regime of the larger guide field (case II) an excessive level of the output power was computed in the three-dimensional simulation [16]. Our 1.5D simulation showed the same discrepancy. According to [26], the amplification is reduced in the regimes with a positive guide field that is caused by the competition between the operating wave and high-frequency modes excited from the noise level. Such processes were not included both in the ARACHNE model as well as in our 1.5D model.

The spatial power distribution obtained by the $1.5 \mathrm{D}$ code for the case of the reversed guide field (III) is plotted in Fig. 1. Experimental data are shown with dots.

One can see reasonable agreement between the power growth calculated using the $1.5 \mathrm{D}$ model and the experimental and 3D simulation results within the starting and linear stages of the amplification. Nevertheless, the power during the saturation stage has been obviously underestimated. The reason lies in the influence of the amplified electromagnetic wave on the nature of transverse motion of the particles. The deviation from their steady helical orbits is expected to be not of helical character but more complicated.

To confirm this explanation, we have analyzed the distributions of the perturbations in the particle velocities versus the phase. The perturbations appear to grow sufficiently for some particles, and already at a distance of about $70 \mathrm{~cm}$ from the entrance of the interaction region, both $\left|\delta \beta_{z}\right|$ and $\left|\delta \beta_{\perp}\right|$ values reach the level of about 0.1 (see the example in Fig. 2). Thus for distances larger than this value the requirements given by (9) and (10) are violated and one can no longer expect the electron trajectories to be pure helical with a small perturbation beyond this point.

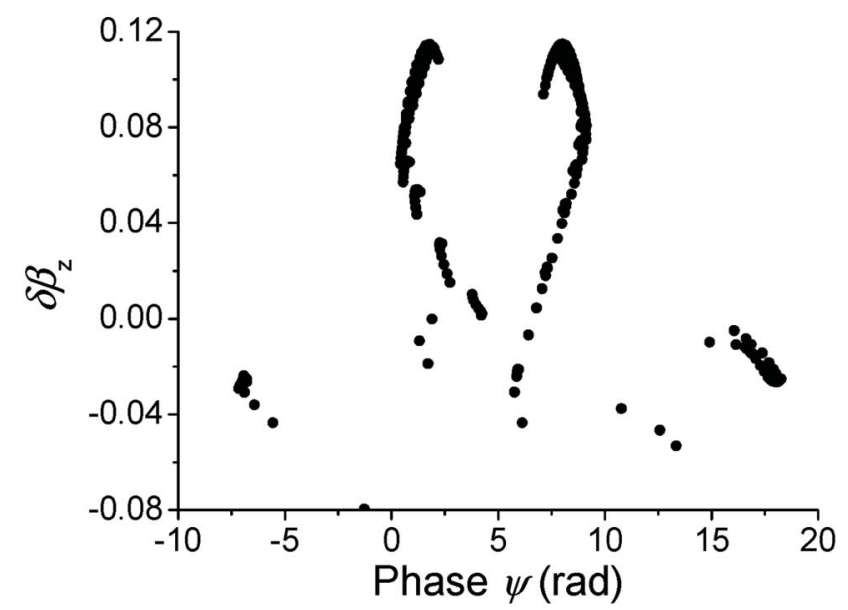

FIG. 2. Distributions of the perturbations of transverse and longitudinal velocities versus phase at $z=135 \mathrm{~cm}$ from the entrance of the interaction region (for the initially single-wavelength section of the beam). 
As it can be illustrated by tracking the phase space of a bunch, the particles, whose motion is not described correctly, fall out of the "trapped" area in the phase space and do not contribute to the pumping of the electromagnetic wave. It is therefore not surprising that the simulation starts to deviate from the experimental results (as well as from the 3D simulation results) at distances larger than about $70 \mathrm{~cm}$ and that the 1.5D simulation underestimates the saturated power.

As azimuthal motion cannot be properly considered within our approach, a three-dimensional consideration could be expedient. It is confirmed by the fact that the three-dimensional simulation [16] (dotted lines in Fig. 1) fits the experimental data substantially better. Virtually all data points fall into two curves corresponding the two sets of parameters lying within uncertainties imposed by the experiment.

The principal difference between our 1.5D code and the 3D code ARACHNE [16] lies in the description of particle dynamics. As for the structure of the electromagnetic and space-charge fields, our model does not contain principal simplification relative to the 3D one. Since the FEL operated in the single-mode amplification regime, only one microwave mode, namely, the $T E_{11}$ mode of cylindrical waveguide, was kept in the both models. The space-charge field, in general, should be considered as a superposition of Gould-Trivelpiece modes of the beam [25]. However, as stated in [16], it is enough to involve only the lowest-order beam mode to give a reasonable agreement with the experiment. In this case the potential term from longitudinal component of Lorentz force equation reduces to that of Eq. (4).

The difficulties in the simulation of the positive-field regimes, especially approaching the cyclotron resonance, confirm the limited applicability of helical-trajectory approach to describe the particle motion. For a more adequate simulation of the amplification process in the FEL, it is expedient to employ full-scale three-dimensional codes. One should foresee the possibility of excitation of microwave parasite modes and take into the account the features of beam delivery to the interaction region and wiggler and solenoid inhomogeneities.

\section{B. FEM amplifier without guide magnetic field at CESTA}

In the experiments at CESTA (France) [14,15], a FEM without a guide magnetic field was investigated as an electron beam buncher. According to the criteria (1) and (2) for space-charge effects (see Table I), the FEM operated in the high-gain Compton regime.

A numerical simulation of microwave amplification and electron beam bunching was performed in [14] with the three-dimensional code SOLITUDE [18]. That code used the same FEL formalism as ARACHNE, with a slightly different numerical method, but with significant differences in the description of the electron beam and in the computation of the space-charge effects. It was benchmarked using the earlier FEL experiments and by comparison with the code ARACHNE.

The experimental data and simulation results from SOLITUDE for the microwave power [14] are plotted with dots and crosses in Fig. 3, respectively. The apparent discrepancy in launching time and saturation power was attributed to an nonideal beam that could not be properly described in the simulation. The differences between the experimental electron beam and the simulated version were attributed to effects of the kicker magnet, adiabatic entrance of the wiggler, and misalignment of the beam [14].

This experiment was also modeled by us using a simplified version of $1.5 \mathrm{D}$ model that assumed a rough approximation for transverse velocities [22]. The results of this model for the power are also shown in Fig. 3 (curve 1) for the case of a cold electron beam (i.e., no energy spread). Rough agreement with simulation [14] was demonstrated.

The result of the current 1.5D model, which allows the transverse velocity to vary as a result of the interaction of the electrons with the microwave field, is shown as curve 2 in Fig. 3. One can see that the obtained results (curve 2 for cold beam) display apparently better agreement with the 3D code SOLITUDE.

Again the validity of the numerical results of the $1.5 \mathrm{D}$ model are checked by evaluating the criteria (9) and (10). The characteristic scales of transverse and longitudinal perturbations in the particle velocities are 0.05 and 0.01 , respectively, while $\beta_{\perp 0} \simeq 0.22$. So the helical-trajectory approach seems to be quite applicable.

In $[14,15]$ the results of experimental investigation of beam bunching are reported. To indicate the bunching

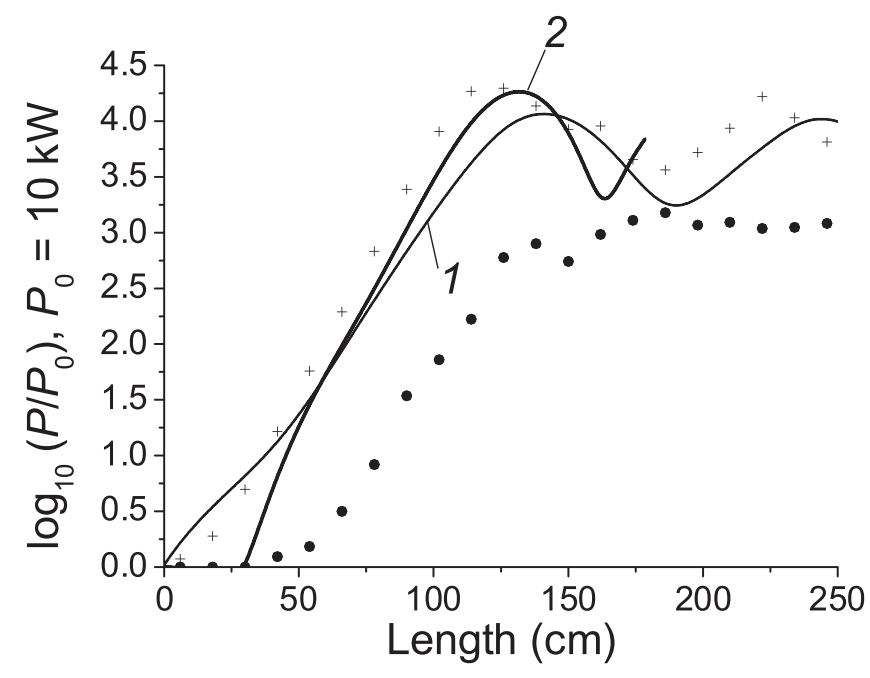

FIG. 3. Power of the CESTA FEM amplifier versus length: CESTA experimental (dots) and simulated (crosses) data [14]; results of simulation for cold beam: 1, from Ref. [22]; 2, by current $1.5 \mathrm{D}$ code. 
quality, one can use the bunching parameter defined as $B=$ $\left|\left\langle e^{i \psi}\right\rangle\right|$ (average over a ponderomotive wavelength). The measured and calculated distributions of $B$ value versus length are shown in Fig. 4 for the different models considered here (cf. Fig. 3). The results of simulation for a cold beam both with 1D code [22] and 1.5D code (curves 1 and 2) are similar to those of [14] (crosses), but they differ significantly from the measured values. According to the authors of [14], this difference is mainly due to the fact that the bunching parameter is measured outside the wiggler. One therefore expects a degradation of the bunching due to longitudinal space charge when the electrons propagate from the end of the wiggler to the position of the bunch length measurement. Note that the distributions of both the power and the bunching parameter obtained with the $1.5 \mathrm{D}$ code are terminated earlier than the data of [14] since we do not consider the adiabatic entrance and exit and simulate the interaction within the regular part of the wiggler only.

\section{FEM amplifier with reversed guide magnetic field at JINR}

In the late 1980s, a FEM amplifier with a reversed guide magnetic field and based on the LIU-3000 linac was investigated experimentally at JINR. The basic results are given in $[7,8]$.

Evaluation of the conditions (1) and (2) for the parameters of this experiment [7] shows that this experiment operates at the boundary between the high-gain Compton regime and the Raman regime.

The dispersion relation at nonzero guide magnetic field is shown in [4] under assumption of small transverse electron velocities and normalized amplitude of the radia-

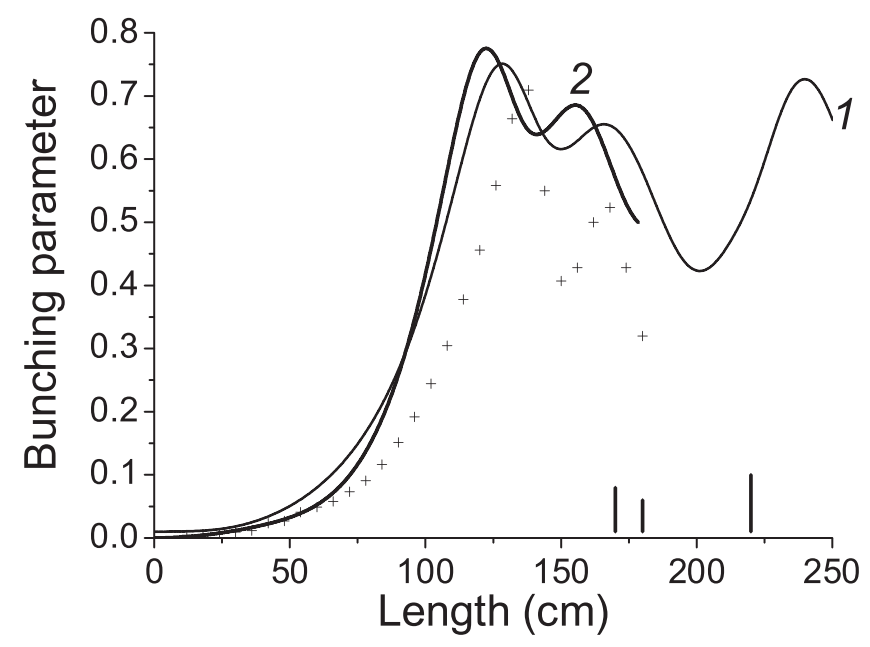

FIG. 4. Bunching parameter of the CESTA FEM amplifier versus length: CESTA experimental (vertical lines) and simulated (crosses) data [14]; results of simulation for cold beam: 1, from Ref. [22]; 2, by current $1.5 \mathrm{D}$ code. tion wave small compared to the normalized amplitude of the wiggler field. It allows one to transform Eq. (5) into

$$
\frac{d \theta_{j}}{d Z}=\frac{c\left(k_{s}+k_{w}\right)}{\omega_{0}}-\frac{1}{\beta_{z j}}-\frac{\omega_{p} \sqrt{\Phi_{0}}}{\omega_{0} \beta_{z 0}} .
$$

In a previous publication [27] this experiment was modeled assuming the above mentioned restrictions as well as assuming that space-charge effects could be neglected (Compton regime). Here we use the less restrictive 1.5D model that includes space-charge effects to model the same experiment.

Figure 5 presents the results of the power calculations with the 1.5D code assuming an energy spread of $\delta \gamma / \gamma=$ $2 \%$ without space charge (solid line) and with space charge (dashed line). The measured values for the power as a function of distance within the wiggler are given by the squares. From this we observe that space-charge effects are not dominantly present and that good agreement is obtained between the simulations and experimental data.

The experimental parameters for this experiment were again used to evaluate the criteria (9) and (10), and it was found that the perturbations of the helical trajectories were similar in magnitude as found for the CESTA experiment while $\beta_{\perp 0} \simeq 0.32$. Thus the experimental parameters do not violate the assumptions of the $1.5 \mathrm{D}$ model, and good agreement between the model and experiment is expected and found.

The developed 1.5D code is considered to be a convenient tool to evaluate experiments with a FEM amplifier in the future. Besides, the performed numerical study is of particular interest for exploring operating peculiarities of the FEM oscillator with a Bragg resonator, which employs

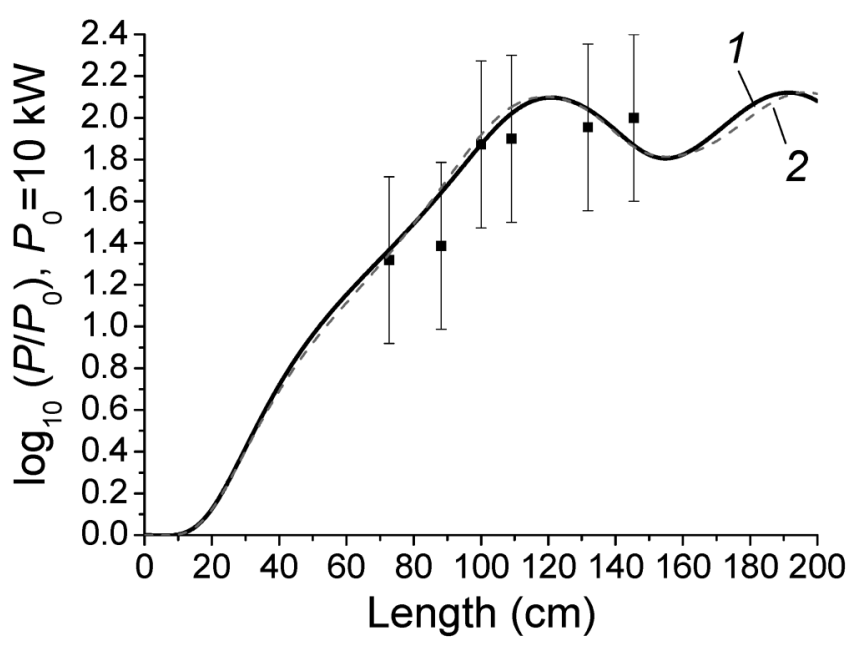

FIG. 5. Power of the FEM amplifier [7] versus length. The experimental data are shown as squares. Results of simulation by $1.5 \mathrm{D}$ code using an energy spread of $\delta \gamma / \gamma=2 \%$ under Raman and Compton models (dashed and solid lines, respectively). 
the beam of the same linac. This facility has been made and studied at JINR jointly with IAP RAS (Nizhny Novgorod) during the last decade $[20,21]$.

\section{CONCLUSIONS}

A 1.5-dimensional model has been composed for FEL amplifiers operating either in the Raman or high-gain Compton regime. The particles are supposed to follow helical trajectories which are computed taking into the account the guide magnetic field, if present, and the variation of particle transverse velocities due to the radiation field and beam self-fields.

As demonstrated in this paper, this model does not require extensive computational resources, but it still takes the dominant physical processes relevant for a FEL amplifier with a helical wiggler into account. The model limitations have been identified and appropriate criteria for them have been formulated.

To prove the usefulness of the model, its results have been compared with both experimental data and results from more elaborate (i.e., three-dimensional) models for a number of millimeter-wavelength FELs (FEMs) $[7,11,14,15]$. These devices operate in different regimes of space-charge and wiggler-guide magnetic field configuration.

For a Raman FEM amplifier [11] with a reversed guide magnetic field, the model predicts the growth of the output power with distance reasonable well as long as saturation is not obtained. At high power levels of the radiation field, it was found that the assumption of pure helical electron orbits with small perturbations breaks down and the model starts to underestimate the output power. For the case of a FEM operating in the high-gain Compton regime without a guide magnetic field and that was used as an electron beam buncher [14,15], good agreement was found between the 1.5D model and a full 3D model of the FEM. The discrepancy between the theoretical simulations and experimental data was the same for the $1.5 \mathrm{D}$ and the $3 \mathrm{D}$ model. For another FEM experiment with a reversed guide magnetic field configuration [7], it was found space-charge effects were still not important despite the fact that the evaluation criteria put the experiment on the border of high-gain Compton and Raman regimes. In this case, good agreement was obtained between the 1.5D model and the experimental data. For the last two experiments it has been verified that the experimental conditions did not violate the assumption of helical electron orbits with small perturbations.

The $1.5 \mathrm{D}$ model has been successfully benchmarked against the 3D models ARACHNE [15] and SOLITUDE [18] and to a lesser extent against experimental data from FEL systems having different operating regimes. The model is useful for a quick and relatively accurate evaluation of the physics of FEL amplifiers having a helical wiggler in combination with a reversed guide magnetic field (that may be zero). It can also easily be extended to study FEL oscillators with similar configurations.

\section{ACKNOWLEDGMENTS}

This work is supported by Grant No. 03-02-16530 of the Russian Foundation for Basic Research.

[1] V.L. Bratman, N. S. Ginzburg, and M. I. Petelin, Opt. Commun. 30, 409 (1979).

[2] T. C. Marshall, Free Electron Lasers (McMillan, New York, 1985).

[3] T. M. Antonsen and H.P. Freund, Principles of FreeElectron Lasers (Chapman \& Hall, London, 1986); or ibid. (Kluwer Academic, Dordrecht, 1996), 2nd ed., ISBN 0-412-72540-1.

[4] C. W. Roberson and P. Sprangle, Phys. Fluids B 1, 3 (1989).

[5] T. J. Orzechowski et al., IEEE J. Quantum Electron. 21, 831 (1985).

[6] T. J. Orzechowski et al., Nucl. Instrum. Methods Phys. Res., Sect. A 250, 144 (1986).

[7] Yu. B. Viktorov et al., in Proceedings of the XI All-Union Seminar on Charged Particle Accelerators, Dubna, 1989 (Joint Institute for Nuclear Research, Dubna, 1989), Vol. II, p. 95 (in Russian).

[8] A.A. Kaminsky et al., Particle Accelerators 33, 189 (1990).

[9] J. S. Wurtele, G. Bekefi, R. Chu, and K. Xu, Phys. Fluids B 2, 401 (1990).

[10] D. A. Kirkpatrick et al., Phys. Fluids B 1, 1511 (1989).

[11] M.E. Conde and G. Bekefi, Phys. Rev. Lett. 67, 3082 (1991).

[12] M. E. Conde and G. Bekefi, Nucl. Instrum. Methods Phys. Res., Sect. A 318, 109 (1992).

[13] P. Zambon, W. J. Witterman, and P. J. M. Van der Slot, Nucl. Instrum. Methods Phys. Res., Sect. A 341, 88 (1994).

[14] J. Gardelle, J. Labrouche, G. Marchese, J. L. Rullier, D. Villate, and J. T. Donohue, Phys. Plasmas 3, 4197 (1996).

[15] J. Gardelle, J. Labrouche, and J. L. Rullier, in Proceedings of the 1996 European Particle Accelerator Conference, Barcelona, 1996, Vol. 1, p. 298.

[16] A. K. Ganguly and H. P. Freund, Nucl. Instrum. Methods Phys. Res., Sect. A 331, 501 (1993).

[17] H.P. Freund, Nucl. Instrum. Methods Phys. Res., Sect. A 331, 496 (1993).

[18] J. Gardelle, J. Labrouche, and P. Le Taillander, Phys. Rev. E 50, 4973 (1994).

[19] Y. Pinhasi, Y. Lurie, A. Yahalom, and A. Abramovich, Nucl. Instrum. Methods Phys. Res., Sect. A 483, 510 (2002).

[20] N. S. Ginzburg et al., Phys. Rev. Lett. 84, 3574 (2000).

[21] A. V. Elzhov et al., in Proceedings of the 2004 Free Electron Laser Conference, Trieste, Italy, 2004 (Sincrotrone Trieste, Trieste, 2004), p. 318.

[22] E. A. Perelstein, L. V. Bobyleva, A. V. Elzhov, and V.I. Kazacha, in Proceedings of the 1997 Particle Acceleration 
Conference, Vancouver, 1997 (IEEE, New York, 1997), p. 488.

[23] N. S. Ginzburg and A. S. Sergeev, Zhurnal Tekhnicheskoi Fiziki. 61, 133 (1991).

[24] H.P. Freund, S. Johnston, and P. Sprangle, IEEE J. Quantum Electron. 19, 322 (1983).
[25] A. K. Ganguly and H. P. Freund, Phys. Fluids 31, 387 (1988).

[26] A. T. Lin, C. C. Lin, and K. R. Chu, Appl. Phys. Lett. 60, 292 (1992).

[27] E. A. Perelstein, L. V. Bobyleva, A. V. Elzhov, and V. I. Kazacha, Nucl. Phys. Investigations 29-30, 78 (1997). 